\title{
Near-wall hindered diffusion: Implications for surface-based sensors
}

\author{
Enno Kätelhön, Stanislav V. Sokolov, Richard G. Compton* \\ Department of Chemistry, Physical and Theoretical Chemistry Laboratory, Oxford \\ University, South Parks Road, Oxford OX1 3QZ, United Kingdom
}

\begin{abstract}
Brownian motion of nanoscale analytes near interfaces is a crucial requirement for the development of numerous surface-based sensors, especially for the detection of individual freely-diffusing particles such as enzymes, proteins, viruses, or nanoparticles. The modelling of diffusion processes near surfaces is much complicated by the hydrodynamic effect of near-wall hindered diffusion of unbound particles at liquid-solid interfaces resulting in anisotropic diffusion at the boundary. We model Brownian motion under the spatially-anisotropic conditions evolving from near-wall hindered diffusion. This reveals detailed insights into the stochastic processes related to Brownian motion and is a new methodology for the modelling of interface-based sensors answering essential questions including the stochastics of the mass transport towards detection sites, average residence times in regions of interest as well as related first passage problems, and power spectral densities of the sensor response.
\end{abstract}

Keywords: Random walks, near-wall hindered diffusion, nano-impacts, nanoparticle collisions

\footnotetext{
* Corresponding author

Email address: richard.compton@chem.ox.ac.uk (Richard G. Compton)
}

Preprint submitted to Sensors and Actuators: B. Chemical

April 13, 2016 


\section{Introduction}

Brownian motion is the key transport mechanism in numerous sensing applications, while particularly methods involving physiochemical surface effects often rely on the diffusion of the analyte towards the site of the detection. Such methods for instance include electrochemical detection, dielectric spectroscopy, surface-plasmon resonance (SPR), surface-enhanced Raman spectroscopy (SERS), or detection via total-internal reflection fluorescence (TIRF), to just name a few.

While diffusive processes in isotropic media can easily be modelled by solving the (isotropic) diffusion equation or via random walks that feature fixed temporal and spatial step widths, the simulation as well as the mathematical description of such transport phenomena is complicated by the classic hydrodynamic effect of near-wall hindered diffusion if the diffusing object is located near a boundary. The particle diffusion coefficient then decreases with distance from the interface, while the deceleration of the Brownian motion differs for diffusion parallel or perpendicular to the boundary. The understanding as well as the ability to measure and model such diffusive mass transport mechanism is hence crucial for the development of the above-mentioned techniques as well as for numerous applications in micro- and nano fluidics, and has therefore been again in the focus of several more-recent studies. $[1,2,3,4]$

As well-established analytical expressions [5, 6] are known for the effect of near-wall hindered diffusion on the diffusion coefficient, the anisotropic diffusion equation can be solved numerically in order to yield for instance average concentration profiles or average rates for the number of diffusing objects arriving at an adsorbing surface. While this information may be sufficient to model sensors that simultaneously detect large numbers of analyte entities, stochastic information such as the systems noise characteristics, which may be of utmost importance in single molecule-[7, 8] or single particle[9] detection, cannot be derived from solving diffusion equation, but requires either the inclusion of appropriate stochastic models[10, 11] or random walk $[8,12,13,14]$ simulations. The answers to the complex stochastic problems that arise in single particle 
detection can hence not be found by numerical solutions of the anisotropic diffusion equation but require new approaches that are capable of modelling the random movements of every single analyte object and thus provide insight into the behaviour of the whole stochastic ensemble.

In this study, we introduce a novel random walk approach for the modelling of Brownian motion under the influence of near-wall hindered diffusion. The presented method exhibits excellent agreement with numerical solutions of the respective diffusion equation for up to 10,000 random walk iterations and allows the detailed simulation of stochastic processes related to diffusive movements near boundaries. As the understanding of such stochastic processes is generally of crucial importance in the development of single enzyme, protein, or nanoparticle sensors that rely on active surfaces as detection sites, the presented method will vitally unpin and promote various fields of research. Applications

likely include the modelling of the statistics of the number of individual analyte molecules or particles impacting on the sensor surface, the distribution of residence times at active sites, the modelling of first passage problems relating to the detection or annihilation of individual analyte entities, and the modelling of the power spectral density of the sensor response.

\section{Theory}

\subsection{Theoretical model}

We consider a one-dimensional geometry comprising diffusing particles near a reflecting boundary at $x=0$. The particle movement can be described via the diffusion equation, which in its most general form is given by:

$$
\begin{aligned}
\frac{\partial c}{\partial t}(x, t) & =\frac{\partial}{\partial x}\left(D \frac{\partial c}{\partial x}\right)(x, t) \\
& =\frac{\partial D}{\partial x} \frac{\partial c}{\partial x}(x, t)+D \frac{\partial^{2} c}{\partial x^{2}}(x, t)
\end{aligned}
$$

where $c$ represents the concentration of particles in solution or the probability of finding a single particle at a certain position, $[10,11] x$ is the distance between 
the boundary and the surface of the particle, and $D(x)$ is the diffusion coefficient as a function of $x$.

Due to the proximity to the boundary, particles experience the effect of near-wall hindered diffusion, which lowers the diffusion coefficient in parallel and perpendicular to the interface. The diffusion coefficient $D(x)$ describing the diffusive motion perpendicular to the boundary differs from the bulk diffusion coefficient $D_{b u l k}$ as discussed by Brenner[6], who derived an expression in form of an infinite series for the reduction of the diffusion coefficient that can be well approximated $[15]^{3}$ by:

$$
D(x)=\lambda(x) \cdot D_{b u l k}=\frac{6 x^{2}+2 x r}{6 x^{2}+9 x r+2 r^{2}} D_{b u l k}
$$

where $D_{b u l k}$ is the bulk diffusion coefficient and $r$ is the particle radius and $x$ the distance between the particle and the surface. This expression was derived for a spherical particle approaching an ideally flat surface. It is however noted that the mass transport of non-spherical particles towards boundaries featuring heterogeneous surface morphologies may either be enhanced or restrained depending on the respective geometry. The here-used model for the diffusion tensor's anisotropy provides a good approximation for diffusion phenomena found under experimental conditions for larger particles, as it was previously shown by Banerjee and Kihm for the particle radii 250 and $500 \mathrm{~nm} \mathrm{[16]} \mathrm{and} \mathrm{Huang}$ and Breuer for the particle radius $1.5 \mu \mathrm{m}$ [17], though smaller particles of the radii 50 and $100 \mathrm{~nm}$ may additionally be affected by physical effects such as gravitational forces, van der Waals forces, and electro-osmotic forces [16]. Figure 1 illustrates the hinderance of the perpendicular diffusion as a function of the particles distance from the surface and is here calculated for various particle radii ranging from 0 to $20 \mathrm{~nm}$.

\footnotetext{
${ }^{3}$ In their original publication[15] Bevan and Prieve incorrectly describe Equation (2) as an approximation of a infinite series provided by Goldman et al. [5] that describes a similar problem. It is however an approximation of the expression derived by Brenner[6] (Equation (2.19) in the Brenner's original publication).
} 


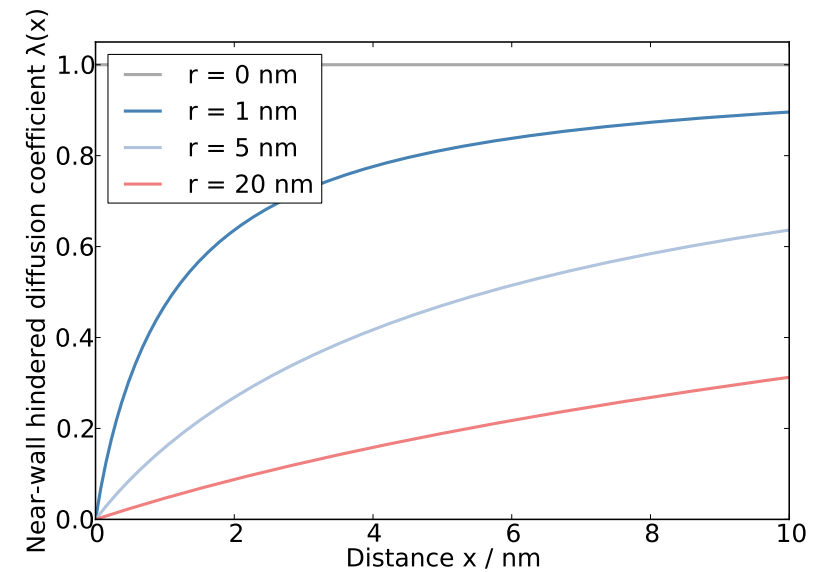

Figure 1: Influence of the effect of near-wall hindered diffusion on the diffusion coefficient as a function of the particle-boundary distance for various particle radii. The diffusion coefficient is given by $D(x)=\lambda(x) D_{b u l k}$.

\subsection{Representation of the random walk}

The above theoretical model can be well approximated by a random walk approach, in which each particle is displaced by a random displacement vector at a constant frequency of $(\Delta t)^{-1}$. Since, in contrast to the isotropic diffusion equation, $\partial_{t} c(t, x)=D \Delta c(t, x)$, the solutions of Equation (1) are not invariant to spatial translations, i.e. they differ depending on the considered particle position in space, [...] we must evaluate the stochastic parameters of each displacement of each random walker individually as a function of its respective momentary position.

In the following, we consider a single particle in the system, the presented approach can however be easily extended to a system of multiple non-interacting particles by calculating different random walks simultaneously and subsequently determining the resulting concentration profile by superposition. After [...] the $\mathbf{i}^{\text {th }}$ random walk step the location $x_{0}(t=i \Delta t)$ [expression amended] of the random walker is exactly determined and the concentration profile can hence be mathematically expressed as: 


$$
c(i \Delta t, x)=\delta\left(x-x_{0}(i \Delta t)\right)
$$

[equation amended] where [...] $\delta(a), a \in \mathbf{R}$, is the Dirac delta function. Starting from this initial concentration profile, we can solve Equation (1) and determine the temporal evolution of the probability distribution of the particle's location within the time interval $\Delta t$. The average displacement $d_{0}\left(x_{0}, \Delta t\right)$ can then be determined by evaluation of the term:

$$
d_{0}\left(x_{0}, \Delta t\right)=\langle x\rangle-x_{0}=\int_{-\infty}^{\infty} d x\left(x-x_{0}\right) c(x, \Delta t)
$$

while the respective average absolute displacement $d_{ \pm}\left(x_{0}, \Delta t\right)$ from the mean displacement can be calculated from the definition of the standard deviation of the particle displacement within the time $\Delta t$ :

$$
d_{ \pm}\left(x_{0}, \Delta t\right)=\sqrt{\left\langle(x-\langle x\rangle)^{2}\right\rangle}=\sqrt{\int_{-\infty}^{\infty} d x(x-\langle x\rangle)^{2} c(x, \Delta t)}
$$

For each random walk step of the length $\Delta t$, we evaluate the expressions (4) and (5) for $d_{0}$ and $d_{ \pm}$, displace the random walker by $d_{0}$, randomly decide at a probability of $p=0.5$ whether the particle moves further away from the boundary or not, and displace it by the respective vector $\pm d_{ \pm}$:

$$
d_{t o t}\left(x_{0}, \Delta t\right)=\left\{\begin{array}{l}
d_{0}\left(x_{0}, \Delta t\right)+d_{ \pm}\left(x_{0}, \Delta t\right) \text { at a probability of } p_{+}=\frac{1}{2} \\
d_{0}\left(x_{0}, \Delta t\right)-d_{ \pm}\left(x_{0}, \Delta t\right) \text { at a probability of } p_{-}=\frac{1}{2}
\end{array}\right.
$$

An illustration of the displacement per random walk iteration can be found in Figure 2. The proposed random walk model hence symmetrically displaces the particle in either direction by the step width $\pm d_{ \pm}$once it was shifted by the average drift $d_{0}$. On the basis of this average displacement $\sigma=d_{ \pm}$(after the shift) that equals the standard deviation around the expected value and the expected value of the particle position after the random walk step $\mu=x_{0}+d_{0}$, we can construct the 
probability density function as a Gaussian distribution. Substitution of $\sigma$ and $\mu$ in the normal distribution reveals that the introduced method approximates [...] the probability distribution of the position of a particle released at the initial position $x_{0}$ before the step via a Gaussian distribution of the form:

$$
p\left(x_{0}, \Delta t\right)=\frac{1}{\sqrt{2 \pi} \cdot d_{ \pm}\left(x_{0}, \Delta t\right)} \exp \left(-\frac{\left(x-x_{0}-d_{0}\left(x_{0}, \Delta t\right)\right)^{2}}{2\left(d_{ \pm}\left(x_{0}, \Delta t\right)\right)^{2}}\right)
$$

[equation amended] Since however the calculation of the random walk via the approximate continuous distribution (7) requires a significantly higher computational effort in comparison to the discrete approach shown in Equation (6), we employ the discrete approach throughout this study.

\section{Computational methods}

Simulations are written in $\mathrm{C}++$ and compiled via the $G N U$ Compiler Collection, and data analysis and visualisation is done in Python using the packages NumPy and matplotlib. The implementations of the finite difference simulation and the random walk simulation are individually detailed below. All results in this work were obtained using free software.

\subsection{Finite difference simulations}

A finite difference approach is employed to solve the diffusion problem stated by Equation (1). To this end, we discretise the equation with respect to a uniform spatiotemporal grid featuring spatial and temporal step widths of $\Delta x$ and $\Delta t$, while the respective grid points are given by:

$$
\begin{aligned}
& x_{i}=i \Delta x \\
& t_{k}=k \Delta t
\end{aligned}
$$






Figure 2: [figure amended] Schematic of a random walk step. During each iteration the random walker is first moved from its initial position $x_{0}(i \Delta t)$ [expression amended] by the average displacement $d_{0}$ to the expected value of the current random walk step, $<x_{0}((i+$ 1) $\Delta t)>$ [expression amended], followed by a second random displacement $d_{ \pm}$to either side. The graphs that are sketched in the background further illustrate the temporal evolution of the probability of finding the particle at a given position. Please note that distances and ratios are not to scale.

and where $i$ and $k$ are the indices of the spatial and temporal steps, respectively. Using a backwards implicit stencil, the diffusion equation (1) can then rewritten as a system of $\left(i_{\max }+1\right)\left(k_{\max }+1\right)$ equations:

$$
\begin{aligned}
\frac{c_{i}^{k}-c_{i}^{k-1}}{\Delta t}= & \frac{D\left(x_{i+1}\right)-D\left(x_{i-1}\right)}{2 \Delta x} \frac{c_{i+1}^{k}-c_{i-1}^{k}}{2 \Delta x} \\
& +D\left(x_{i}\right) \frac{c_{i+1}^{k}-2 c_{i}^{k}+c_{i-1}^{k}}{(\Delta x)^{2}}
\end{aligned}
$$

where $c_{i}^{k}$ substitutes $c(i \Delta x, k \Delta t)$ and $x_{i}=i \Delta x$ for clarity. Rearranging the coefficients, we transform this result into: 


$$
c_{i}^{k-1}=\alpha_{i} c_{i-1}^{k}+\beta_{i} c_{i}^{k}+\gamma_{i} c_{i+1}^{k}
$$

where:

$$
\alpha_{i}=-\frac{\Delta t}{(\Delta x)^{2}}\left(D\left(x_{i}\right)-\frac{\left(D\left(x_{i+1}\right)-D\left(x_{i-1}\right)\right)}{4}\right)
$$

[equation amended] and:

$$
\beta_{i}=\frac{2 \Delta t D\left(x_{i}\right)}{(\Delta x)^{2}}+1
$$

[equation amended] and:

$$
\gamma_{i}=-\frac{\Delta t}{(\Delta x)^{2}}\left(D\left(x_{i}\right)+\frac{\left(D\left(x_{i+1}\right)-D\left(x_{i-1}\right)\right)}{4}\right)
$$

[equation amended] The diffusion problem can then be expressed as a system of $k_{\max }$ tridiagonal sets of equations that are solved for each time step $k$ via the tridiagonal matrix algorithm using the previous time step as the initial condition:

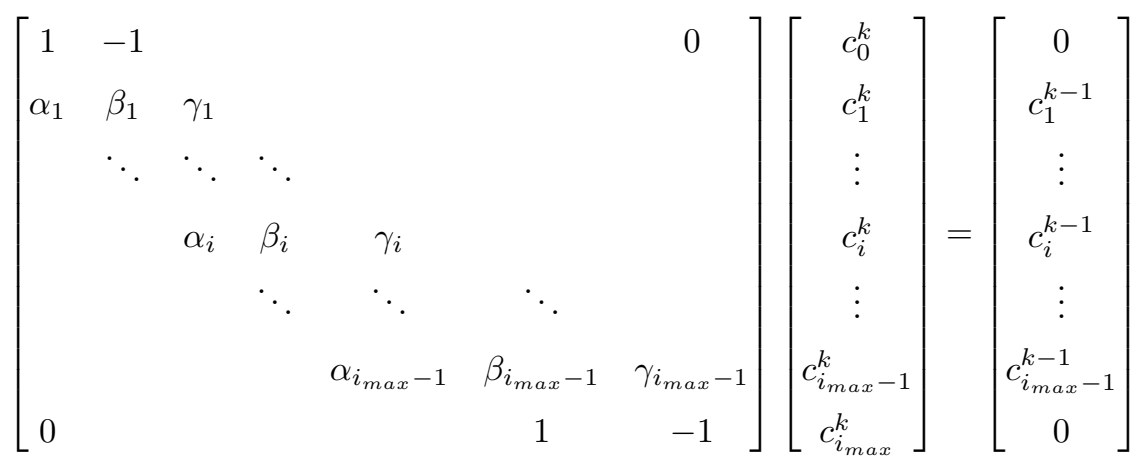

[equation amended] where the equations $c_{0}^{k}=c_{1}^{k}$ and $c_{i_{\max }-1}^{k}=c_{i_{\max }}^{k}$ implement reflecting boundary conditions at either side of the modelled space, $\partial_{x} c(t, x=0)=\partial_{x} c\left(t, x=i_{\max } \Delta x\right)=0$. 


\subsection{Random walk simulations}

We employ random walk simulations on a uniform temporal grid featuring a step width of $\Delta t$. During each random walk step, the random walker is shifted by the vector $d_{0}(x, \Delta t)$ and additionally randomly displaced by the vector $\pm d_{ \pm}(x, \Delta t)$ at a probability of $p=0.5$. As $d_{0}$ and $d_{ \pm}$are both variable and dependent on the particle position, our simulation loads the respective sets of parameters from a previously calculated table and linearly interpolates between the table entries prior to the displacement of the particle during each iteration.

\section{Results and discussion}

We present our results in three subsections that each deal with different aspects of the presented approach. In the first subsection, we discuss the temporal evolution of an initial $\delta$ concentration profile and illustrate how the resultant random walk parameters vary with the choice of the temporal step width $\Delta t$ of the random walk. In the second subsection, random walk parameters are investigated as a function of the position of the random walker for a given $\Delta t$. Last, we compare our random walk simulations with finite difference simulations in order to demonstrate the close agreement of both methods.

All simulations model nanoparticles featuring a radius of $5 \mathrm{~nm}$ and a bulk diffusion coefficient of $10^{-11} \mathrm{~m}^{2} \mathrm{~s}^{-1}$.

\subsection{Temporal evolution of an initial $\delta$-concentration profile}

Using the above-described finite difference approach, we calculate the temporal evolution of a $\delta$-concentration profile that is initially set at the position $x_{\text {init }}=4 \mathrm{~nm}$. The obtained data, which can be seen in Figure 3, demonstrates that the concentration profile broadens over time while exhibiting an almost symmetric profile with respect to the initial position. The calculation of the mean of the distribution $\langle x\rangle$, however, reveals that $\langle x\rangle$ drifts away from the boundary with time as it is indicated by the dashed vertical lines, which at a closer inspection exhibit a small difference. In order to quantify this 


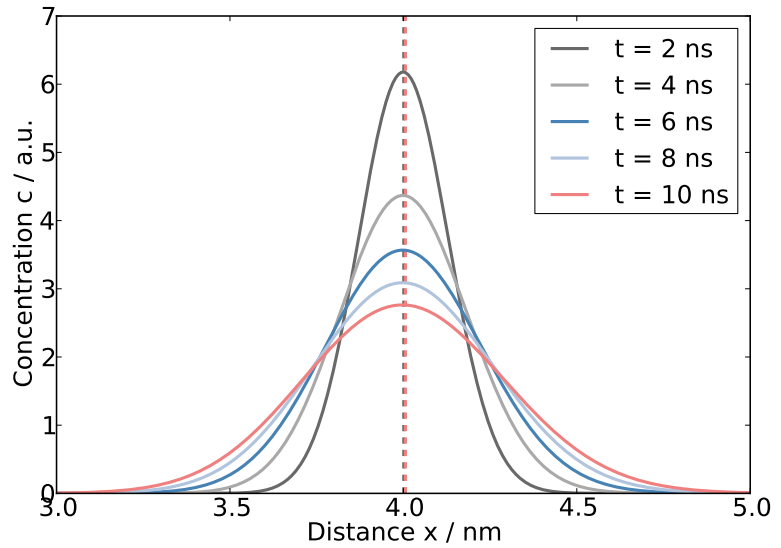

Figure 3: Concentration profile evolving from an initial $\delta$-spike concentration profile located at $x_{\text {init }}=4 \mathrm{~nm}$ as calculated via the finite difference approach. The two almost ideally overlapping dashed vertical lines indicate the means $\langle x\rangle(t)$ of the first and the last depicted concentration profiles.

effect, we do a more rigorous analysis and find that - in contrast to the case of an isotropic diffusion coefficient - the initial symmetric concentration distribution evolves into an increasingly asymmetric distribution over time: Interpreting the distribution as a probability distribution of finding a random walker that was released at $x_{\text {init }}$ at a certain position after the time $\Delta t$, we observe that the probability of moving away from the boundary at $x=0$ differs from the probability of approaching the boundary and that the average displacements in either direction vary from each other, if we evaluate the expressions for the average displacement $d_{0}$ (Equation (4)) and the standard deviation $d_{ \pm}$(Equation (5)). The corresponding data can be found in the Figures 4 and 5, where the plots show that $d_{0}$ as well as $d_{ \pm}$increase over time, clearly demonstrating the asymmetry of the distribution. Comparing these results to linear fits, we can further find that the average displacement increases linearly with $t$ and that the standard deviation grows with the square root of $t$, which corresponds to a linear relation between the variance of the distribution and the duration of the diffusion process. 


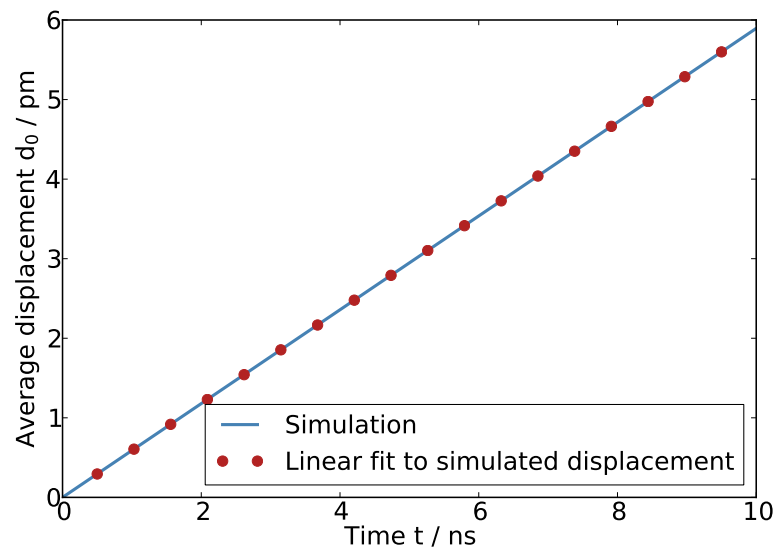

Figure 4: Average displacement of the random walker as a function of time. The displacement was calculated according to Equation (4) from the simulation that is presented in Figure 3. The red dots indicate a linear fit to the simulated data.

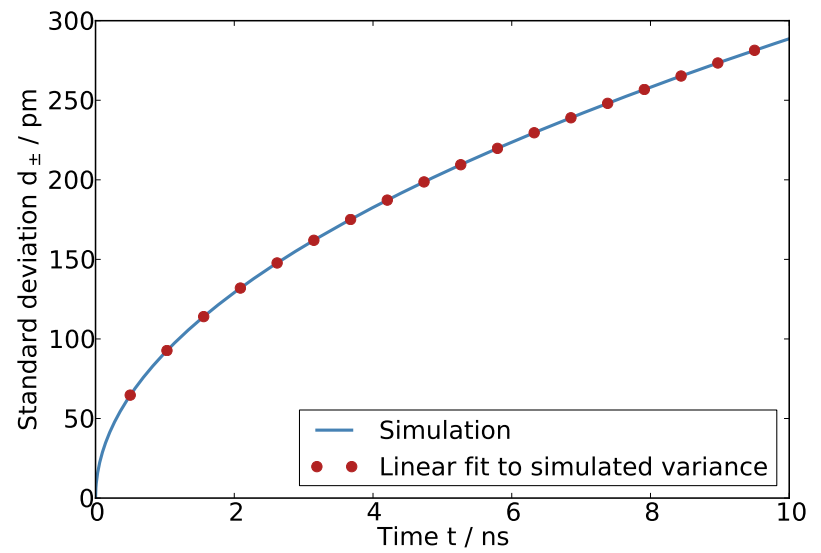

Figure 5: Standard deviation $d_{ \pm}$of the random walker's displacement as a function of time. The standard deviation was calculated according to Equation (5) from the simulation that is depicted in Figure 3. The red dots show a linear fit to the variance of the simulated displacement distribution, $\operatorname{var}=\left(d_{ \pm}\right)^{2}$.

\subsection{Calculation of the random walk parameters}

In order to utilise our finite difference simulation for the development of a random walk that approximates Equation (1), we choose a temporal step width 
$\Delta t \ll t_{\max }$, which is here arbitrarily fixed to $10 \mathrm{ps}$, and calculate the random walker's average displacement after this time interval as well as the respective standard deviation for each point on a uniform one-dimensional grid featuring a pitch of $2 \mathrm{pm}$. To this end, we repeat the process that is described in the previous section at each grid point by modelling the temporal evolution of a delta spike profile that is initially set at the location of the respective grid point and the evaluation of the expressions (4) and (5) for the chosen time interval $\Delta t$. The so-obtained results are presented in the Figures 6 and $7 \mathrm{a}$. In the plots, we can find that the average displacement increases at the boundary, while we find values between 2 and $20 \mathrm{fm}$ within a distance of less than $8 \mathrm{~nm}$ from the boundary for a particle featuring a radius of $5 \mathrm{~nm}$. Due to the increasing diffusion coefficient at larger distances from the boundary, we further find the standard deviation increases with the distance of the release point from the boundary. Here, we calculate values of up to $11 \mathrm{pm}$, which demonstrates that stochastic displacement $d_{ \pm}$significantly exceeds the drift $d_{0}$ in this region.

Figure 7 additionally compares the standard deviation of the here-suggested approach with the standard deviation $d_{ \pm}^{\prime}$ that was used in previously-reported $[9,18]$ random walk simulations including the effect of near-wall hindered diffusion, while assuming the diffusion coefficient to be locally constant within the range of one random walk step. In these simulations, the particle is displaced by a random vector perpendicular to the boundary that features a length of $d_{ \pm}^{\prime}$ and either points towards or away from the boundary at a probability of 0.5 , while $d_{ \pm}^{\prime}$ is calculated as:

$$
d_{ \pm}^{\prime}=\sqrt{2 D\left(x_{0}\right) \Delta t}
$$

where $x_{0}$ is the position of the random walker. In the plots, we find that both approaches lead to almost identical standard deviations, while a more detailed analysis further shows that the differences between the two models amount to less than $0.5 \cdot 10^{-16} \mathrm{~m}$ in the interval $0.5 \mathrm{~nm}<\mathrm{x}<7.5 \mathrm{~nm}$, which is several orders of magnitude below the average displacement $d_{0}$ (data not shown). The 


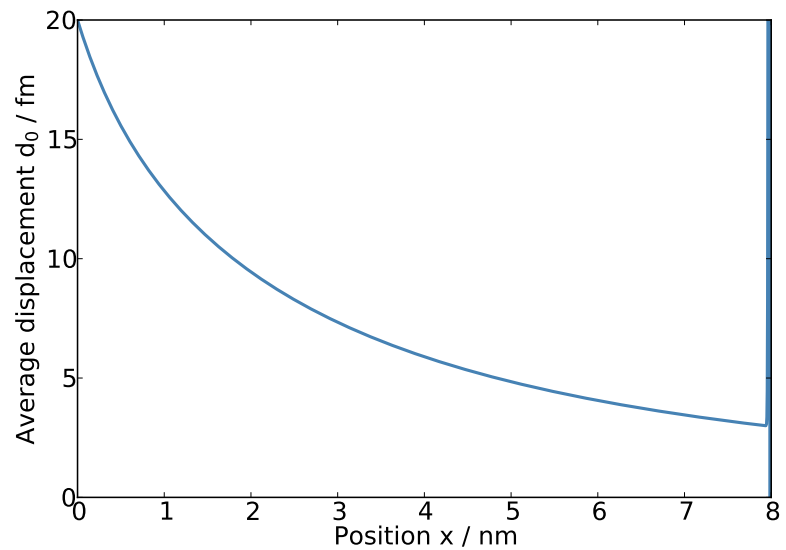

Figure 6: Average displacement of the random walker as a function of its initial position. The data was calculated for a diffusion process within the time interval $\Delta t=10 \mathrm{ps}$.

dominant difference between the previously- and the here-reported approach is hence the inclusion of the additional displacement $d_{0}$. The magnitude of the difference $\Delta\langle x\rangle(t)$ in the average final positions of two random walkers in the two models can therefore be estimated as:

$$
\Delta\langle x\rangle(t) \approx \frac{d_{0}\left(x_{\text {init }}\right)+d_{0}\left(x_{\text {final }}\right)}{2} \frac{t}{\Delta t}
$$

\subsection{Comparison of finite difference and random walk simulations}

We exploit the previously calculated parameters for the average displacement $d_{0}$ and the standard deviation $d_{ \pm}$to set up a random walk simulation. In order to avoid effects arising from the presence of boundaries in the finite difference simulation, we evaluate 200,000 random walks in a distance of $0.5 \mathrm{~nm}$ to 7.5 $\mathrm{nm}$ from the electrode, while reflecting boundaries are set at either side. All random walkers are initially uniformly distributed in the interval between 3.8 $\mathrm{nm}$ and $4.2 \mathrm{~nm}$ from the boundary and are released at $t=0$. In addition to that, we calculate the same model using the finite difference approach for direct comparison. In the finite difference simulation, the initial concentration profile is hence given by: 


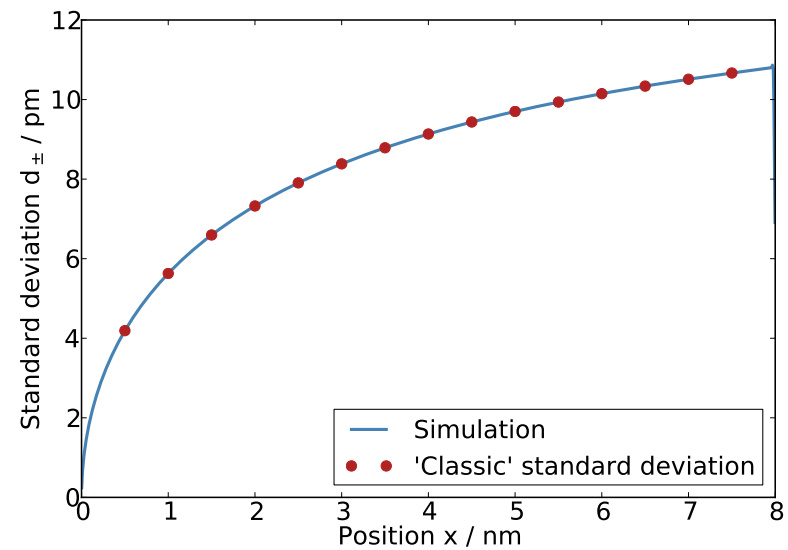

Figure 7: Simulated standard deviation $d_{ \pm}$of the random walker's diffusive displacement as a function of its initial position and after a time interval of $\Delta t=10 \mathrm{ps}$. The data is compared to the standard deviation (15) that was used in previous studies.

$$
c(x, t=0)= \begin{cases}0 & \text { for } 0<x<3.8 \mathrm{~nm} \\ \text { const. } & \text { for } 3.8<x<4.2 \mathrm{~nm} \\ 0 & \text { for } 4.2<x<8 \mathrm{~nm}\end{cases}
$$

The results of both simulations are depicted in Figure 8, where we find almost identical results after 5,000 and 10,000 random walk iterations, which corresponds to 50 and $100 \mathrm{~ns}$, respectively.

In addition to that we compare our approach to above-mentioned and previouslyreported $[9,18]$ simulations. As we saw in Figure 4 the average displacement $d_{0}$ per random walk step is relatively small in comparison to the random displacement $d_{ \pm}$, which is calculated as the standard deviation of the distribution and which does not significantly differ from the previously reported approach. Since random walk simulations may however employ a significant number of random walk steps per random walker, the inclusion of the displacement $d_{0}$ may impact the outcome of the simulation as estimated in Equation (16). In order to illustrate these deviations, we implement a random walk simulation as described by Equation (15) and compare it to a finite difference simulation, see Figure 9. In 


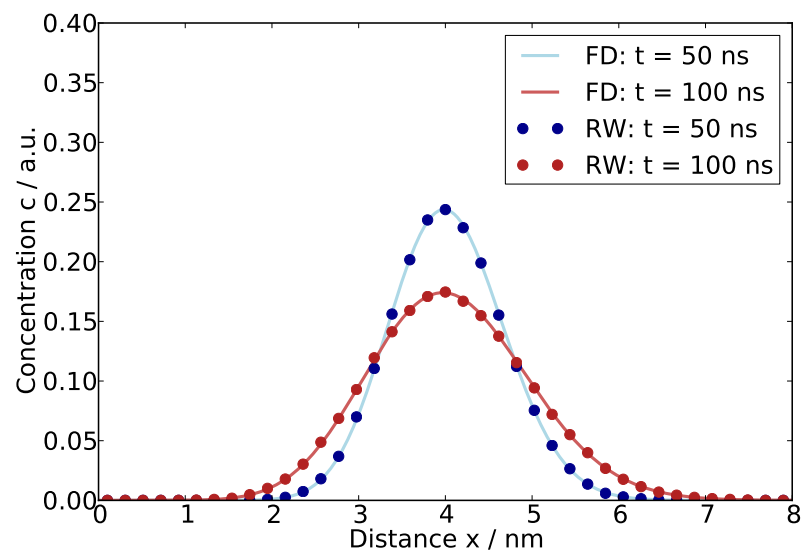

Figure 8: Comparison between concentration profiles that are obtained from a random walk simulation and from a finite difference simulation. The random walk profile shows the result of $2 \cdot 10^{5}$ particles after up to 10,000 random walk iterations using the previously calculated average displacements $d_{0}$ and standard deviations $d_{ \pm}$, see Figures 6 and 7 . At $t=0$, random walkers are uniformly distributed between $3.8 \mathrm{~nm}$ and $4.2 \mathrm{~nm}$.

the plot, we can find the expected deviation of the random walk from the finite difference result. Since individual steps do not include the average displacement of the random walker away from the boundary, the random walkers are in average closer to the electrode. Neglecting the average displacement $d_{0}$ hence leads to an overestimation of the time of residence near the boundary and eventually to an agglomeration of random walkers.

\section{Practical application examples}

[section added] In order to illustrate how our random walk approach can be utilised in sensor development, in the following we provide two practical examples:

- Correlation spectroscopy:

In analogy to fluorescence correlation spectroscopy (FCS), surfacebased sensors can be developed that enable the detection of analyte objects in a locally-confined sensing space. The diffusion of 


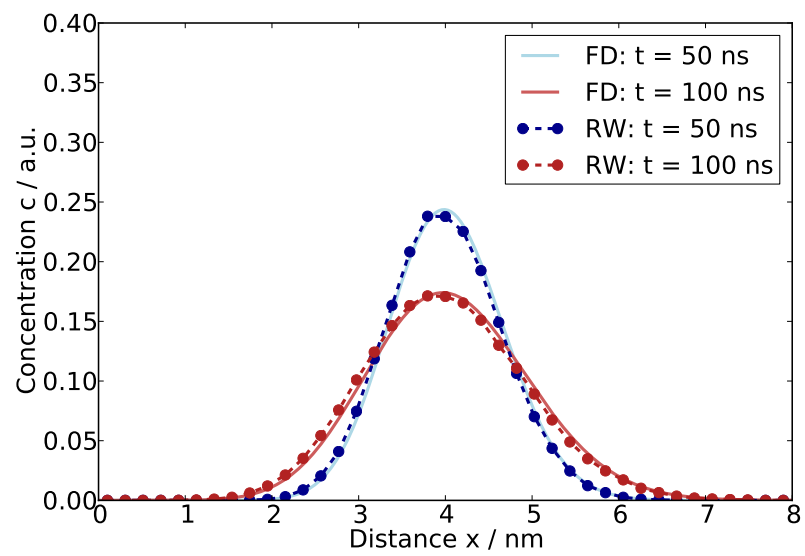

Figure 9: Comparison between concentration profiles that are obtained from a random walk simulation according to Equation (15) and from a finite difference simulation. All parameters equal the parameters that were chosen in Figure 8

the analyte into and out of this sensing area then leads to a characteristic noise signal in the sensor response, which reflects the analyte concentration and its diffusion coefficient. The application of our modelling approach as it is provided in Equation (6) enables the modelling of this sensor response and may provide helpful tool in the development of such sensors.

- Size distributions of analyte populations:

Various types of optical surface-based sensors can detected the presence of freely-diffusing analyte objects near the sensor surface, though the analyte's size distribution cannot be resolved. A detailed analysis of the noise characteristics of the sensor response at a given analyte concentration may however reveal the distribution of the analyte's diffusion coefficients and hence their sizes as smaller objects will exhibit different average times of residence at the surface area compared to larger analytes. The respective noise response can be modelled via random walks in 


\section{accordance with Equation (6), which may provide detailed in- sights into the mass transport of such heterogeneous systems at the sensor interface.}

\section{Conclusions}

We introduce a new rigorous random walk approach for the simulation of Brownian motion near boundaries. Through the addition of a 'drift'-term in the mathematical representation of the random walk, our method novelly recognises the asymmetric temporal evolution of concentration profiles under the spatially anisotropic conditions caused by the effect of near-wall hindered diffusion. Giving broad insights into the diffusive motion at interfaces, the presented approach facilitates the understanding of stochastic effects that directly arise from the analyte's Brownian motion and that are generally of crucial importance in singlemolecule or single-particle sensing approaches relying on surfaces as detection sites, and can be used to elucidate numerous problems such as the stochastics of the mass transport towards detection sites, average residence times in regions of interest as well as related first passage problems, and power spectral densities of the sensor response at a previously-unmatched level of detail. The method will be of great significance for a variety of different fields of research that focus on the interaction between surfaces and individual diffusing objects like nanoparticles, enzymes, proteins, or viruses. [...] We look forward to seeing its application in future studies.

\section{Acknowledgements}

The research leading to these results has received partial funding from the European Research Council under the European Union's Seventh Framework Programme (FP/2007-2013)/ERC Grant Agreement No. [320403]. 


\section{References}

[1] P. Sharma, S. Ghosh, S. Bhattacharya, A high-precision study of hindered diffusion near a wall, Applied Physics Letters 97 (10) (2010) 104101.

[2] Y. Kazoe, M. Yoda, Measurements of the near-wall hindered diffusion of colloidal particles in the presence of an electric field, Applied Physics Letters 99 (12) (2011) 124104.

[3] E. O. Barnes, Y.-G. Zhou, N. V. Rees, R. G. Compton, The effect of near wall hindered diffusion on nanoparticle-electrode impacts: A computational model, Journal of Electroanalytical Chemistry 691 (2013) 28-34.

[4] K. Huang, I. Szlufarska, Effect of interfaces on the nearby brownian motion, Nature Communications 6 (2015) 8558.

[5] A. Goldman, R. G. Cox, H. Brenner, Slow viscous motion of a sphere parallel to a plane wall - i motion through a quiescent fluid, Chemical Engineering Science 22 (4) (1967) 637-651.

[6] H. Brenner, The slow motion of a sphere through a viscous fluid towards a plane surface, Chemical Engineering Science 16 (3) (1961) 242-251.

[7] M. A. Zevenbergen, P. S. Singh, E. D. Goluch, B. L. Wolfrum, S. G. Lemay, Stochastic sensing of single molecules in a nanofluidic electrochemical device, Nano Letters 11 (7) (2011) 2881-2886.

[8] P. S. Singh, E. Kätelhön, K. Mathwig, B. Wolfrum, S. G. Lemay, Stochasticity in single-molecule nanoelectrochemistry: Origins, consequences, and solutions, ACS Nano 6 (11) (2012) 9662-9671.

[9] E. Kätelhön, R. G. Compton, Understanding nano-impacts: impact times and near-wall hindered diffusion, Chemical Science 5 (12) (2014) 4592-4598.

[10] S. Eloul, E. Kätelhön, C. Batchelor-McAuley, K. Tschulik, R. G. Compton, Diffusional nanoimpacts: The stochastic limit, The Journal of Physical Chemistry C 119 (25) (2015) 14400-14410. 
[11] S. Eloul, E. Kätelhön, C. Batchelor-McAuley, K. Tschulik, R. G. Compton, Diffusional impacts of nanoparticles on microdisc and microwire electrodes: The limit of detection and first passage statistics, Journal of Electroanalytical Chemistry 755 (2015) 136-142.

[12] E. Kätelhön, K. J. Krause, P. S. Singh, S. G. Lemay, B. Wolfrum, Noise characteristics of nanoscaled redox-cycling sensors: Investigations based on random walks, Journal of the American Chemical Society 135 (24) (2013) $8874-8881$.

[13] E. Kätelhön, K. J. Krause, K. Mathwig, S. G. Lemay, B. Wolfrum, Noise phenomena caused by reversible adsorption in nanoscale electrochemical devices, ACS Nano 8 (5) (2014) 4924-4930.

[14] C. Batchelor-McAuley, E. Kätelhön, E. O. Barnes, R. G. Compton, E. Laborda, A. Molina, Recent advances in voltammetry, ChemistryOpen 4 (3) (2015) 224-260.

[15] M. A. Bevan, D. C. Prieve, Hindered diffusion of colloidal particles very near to a wall: Revisited, The Journal of Chemical Physics 113 (3) (2000) $1228-1236$.

[16] A. Banerjee, K. D. Kihm, Experimental verification of near-wall hindered diffusion for the brownian motion of nanoparticles using evanescent wave microscopy, Physical Review E 72 (4) (2005) 042101.

[17] P. Huang, K. S. Breuer, Direct measurement of anisotropic near-wall hindered diffusion using total internal reflection velocimetry, Physical review E 76 (4) (2007) 046307.

[18] M. Kang, D. Perry, Y.-R. Kim, A. W. Colburn, R. A. Lazenby, P. R. Unwin, Time-resolved detection and analysis of single nanoparticle electrocatalytic impacts, Journal of the American Chemical Society 137 (34) (2015) 1090210905. 